This is the author's final, peer-reviewed manuscript as accepted for publication. The publisher-formatted version may be available through the publisher's web site or your institution's library.

\title{
An accurate and portable solid state neutron rem meter
}

T. M. Oakes, S. L. Bellinger, W. H. Miller, E. R. Myers, R. G. Fronk, B. W. Cooper, T. J. Sobering, P. R. Scott, P. Ugorowski, D. S. McGregor, J. K. Shultis, and A. N. Caruso

\section{How to cite this manuscript}

If you make reference to this version of the manuscript, use the following information:

Oakes, T. M., Bellinger, S. L., Miller, W. H., Myers, E. R., Fronk, R. G., Cooper, B. W., ... Caruso, A. N. (2013). An accurate and portable solid state neutron rem meter.

Retrieved from http://krex.ksu.edu

\section{Published Version Information}

Citation: Oakes, T. M., Bellinger, S. L., Miller, W. H., Myers, E. R., Fronk, R. G., Cooper, B. W., ... Caruso, A. N. (2013). An accurate and portable solid state neutron rem meter. Nuclear Instruments and Methods in Physics Research A, 719, 6-12.

Copyright: (C) 2013 Elsevier B.V.

Digital Object Identifier (DOI): doi:10.1016/j.nima.2013.03.060

Publisher's Link: http://www.sciencedirect.com/science/article/pii/S0168900213003707

This item was retrieved from the K-State Research Exchange (K-REx), the institutional repository of Kansas State University. K-REx is available at http://krex.ksu.edu 


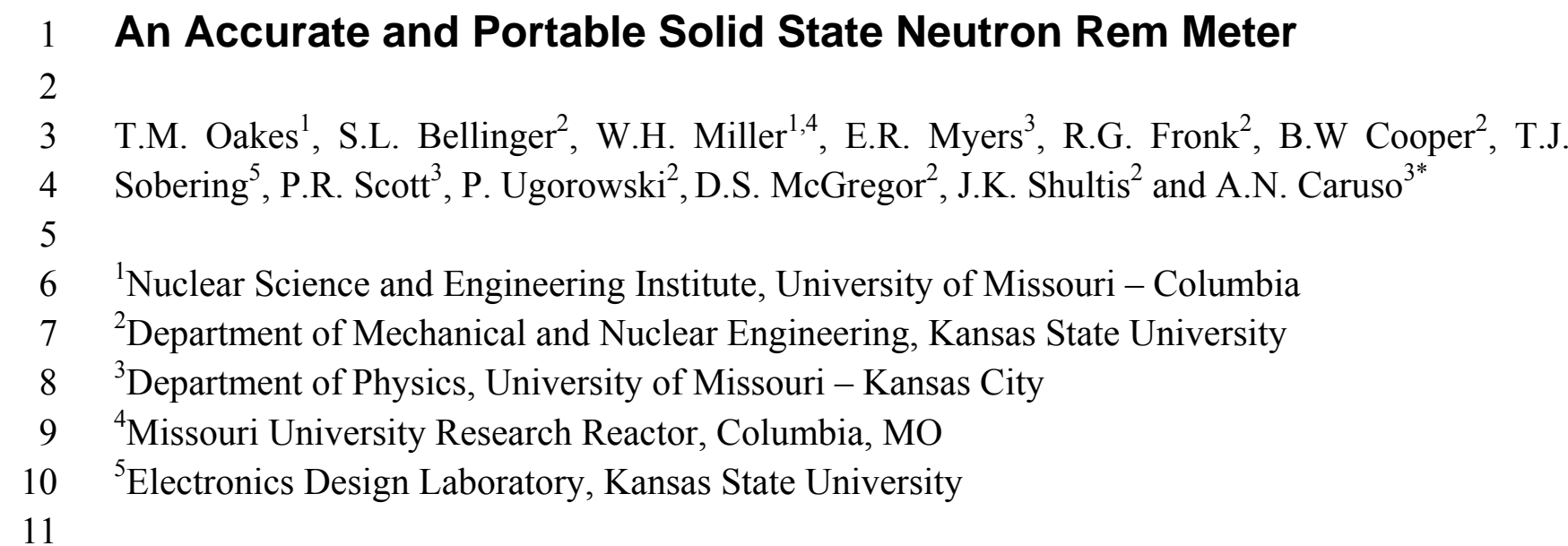

12 Keywords: neutron dosimeter, solid state neutron detector, spectrometer

\section{Abstract}

Accurately resolving the ambient neutron dose equivalent spanning the thermal to $15 \mathrm{MeV}$ energy range with a single configuration and lightweight instrument is desirable. This paper presents the design of a portable, high intrinsic efficiency, and accurate neutron rem meter whose energy-dependent response is electronically adjusted to a chosen neutron dose equivalent standard. The instrument may be classified as a moderating type neutron spectrometer, based on an adaptation to the classical Bonner sphere and position sensitive long counter, which, simultaneously counts thermalized neutrons by high thermal efficiency solid state neutron detectors. The use of multiple detectors and moderator arranged along an axis of symmetry (e.g., long axis of a cylinder) with known neutron-slowing properties allows for the construction of a linear combination of responses that approximate the ambient neutron dose equivalent. Variations on the detector configuration are investigated via Monte Carlo N-Particle simulations to minimize the total instrument mass while maintaining acceptable response accuracy - a dose error less than $15 \%$ for bare ${ }^{252} \mathrm{Cf}$, bare $\mathrm{AmBe}$, an epi-thermal and mixed monoenergetic sources is found at less than $4.5 \mathrm{~kg}$ moderator mass in all studied cases. A comparison of the energy dependent dose equivalent response and resultant energy dependent dose equivalent error of the present dosimeter to commercially-available portable rem meters and the prior art are presented. Finally, the present design is assessed by comparison of the simulated output resulting from applications of several known neutron sources and dose rates.

\section{Submission: Nuclear Instruments and Methods in Physics Research A}

Version: 25 FEB 2013

*Corresponding Author: Anthony N. Caruso, carusoan@umkc.edu, 816-235-2505 


\section{Introduction}

41 The first wide-energy range instrument for measuring neutron dose equivalent was introduced in

421962 by D.E. Hankins [1]. The Hankins moderating instrument, building on the ten-inch Bonner

43 sphere response [2], was a paradigm shifting technology in neutron dose equivalent metrology in

44 that the energy dependent dose equivalent [3-5] from thermal to ones-of-MeV could be

45 approximated without directly measuring the neutron energy spectrum.

47 Since the mid-1960s, five classes of wide-energy range neutron dosimeters have emerged in an

48 effort to improve: (1) the accuracy of measured quantities proportional to neutron energy; (2) the

49 intrinsic detection efficiency; (3) the instrument mass; and/or (4) to extend the neutron energy

50 range. These classes include: single or multiple detectors enclosed by single or multiple neutron

51 interaction materials. In the first class, a combination of boron and/or cadmium, lead or tungsten,

52 and high hydrogen concentration material (usually, high density polyethylene, or HDPE) are

53 used as filters, spallation centers, and moderators to provide ever better response to the dose

54 equivalent curve at up to ones-of-GeV incident neutron energy (e.g., Canberra's SNOOPY or

55 Thermo's SWENDI-II) [6-12]. These instruments are known colloquially as the Andersson-

56 Braun (AB) type. The downside of this approach is that the total mass is high (usually $>10 \mathrm{~kg}$ )

57 and the intrinsic detection efficiency is low $(0.25 \%$ and $0.05 \%$ for the SWENDI-II and SNOOPY

58 respectively, in response to bare ${ }^{252} \mathrm{Cf}$ ). In the second case, multi-band detectors usually tune

59 three or more detectors to the thermal, epithermal, and fast neutron spectrum ranges of the dose

60 equivalent curve using filtering techniques but without extraneous moderator [13-18]. The

61 implication is a lightweight dose equivalent meter (e.g., Ludlum's PRESCILA) but the average

62 dose- and dose-rate error over the thermal to fast range is consequently the highest of the five 
63 methods because of severe over or under response in the bands not covered. The third method

64 employs many individual thermal neutron detectors in an HDPE or comparable moderating

65 matrix to provide a depth dependent intensity of thermalized neutrons that yields both the highest

66 efficiency and lowest average dose- and dose-rate-error of the above methods [19-27]. The

67 shortfall of these instruments is their large moderating volume (usually a $30 \mathrm{~cm}$ diameter sphere)

68 needed to accommodate tens-to-hundreds of individual detectors, rendering a non-portable

69 device ( $>18 \mathrm{~kg}$ with electronics). The fourth method utilizes a single position sensitive detector

70 enclosed by moderator and filter materials as an improvement to the classical long counter [28-

$7130]$. Although simple, this detection scheme suffers from large moderating volumes and low

72 intrinsic efficiency due to high neutron absorption in the moderator and/or scattering of neutrons

73 outside the detector volume. There are only a few examples of the fifth class which utilize a

74 combination of elements from the first three [31-35]. Like the second class, these dosimeter

75 schemes use a superposition of responses to better approximate the dose equivalent curve, but

76 they incorporate an important improvement in that the overlapping energy response bands are

77 continuous. This provides for a much better dose equivalent match, even up to ones-of-GeV,

78 than that available commercially. The downside is, again, the large total volume and low

79 intrinsic efficiency. Neither the third, fourth, nor fifth device classes have been adopted for

80 commercial production.

82 Due to their minimum size requirements, the continued use of gaseous- and scintillator-based

83 thermal neutron detectors in wide-energy, moderating-type neutron dosimeters perpetuates an

84 intrinsic tradeoff between dose error (closest match to the dose equivalent curve), volume of

85 moderator needed, and total detection efficiency (or time/fluence needed to attain reasonable 
86 statistics). For the moderating-type classes given above, these tradeoffs can be lessened via a

87 cross-over to solid state methods of neutron detection that allow for a reduced perturbation to the

88 neutron slowing down process (i.e. increased spatial detection resolution) as well as

89 enhancements to intrinsic efficiency. The reduced perturbation stems from the ability to fabricate

90 devices (p-n junctions) nearly wafer thin $(<500 \mu \mathrm{m})$ while retaining high intrinsic efficiency. The

91 high intrinsic efficiency is derived from both the high thermal detection efficiency capabilities

92 (described elsewhere [37]) as well as the detector-moderator geometry (i.e., minimizing neutron

93 absorption in the moderator). The work reported here describes a significantly improved method

94 for measuring the ambient neutron dose equivalent through a combination of superposed

95 detectors and electronic response matching to the dose equivalent standard [38]. The result is a

96 portable instrument that is adjustable to any dose equivalent quantity, but still retains high

97 intrinsic efficiency, and low dose equivalent error for neutrons with energy less than $15 \mathrm{MeV}$.

98

\section{2. Design Philosophy}

100 The operational quantity devised by the International Commission on Radiation Units and 101 Measurement (ICRU) for operational radiation field measurements is the ambient dose 102 equivalent, $H^{*}(10)$, which represents the dose equivalent at a point of interest in a radiation field 103 which would be generated at a $10 \mathrm{~mm}$ depth in a superimposed tissue-equivalent sphere [39]. For 104 the case of mono-energetic neutrons at energy $E$, the ambient dose equivalent can be determined 105 by

106

$$
H^{*}(10)=\Phi h_{c c, E}
$$


108 where $\Phi$ is the mono-energetic neutron fluence and $h_{c c, E}$ is a neutron dose-equivalent conversion

109 value specific to the energy of the incident neutrons that accounts for both the quantity of energy

110 absorption and the corresponding relative biological effects (Fig. 1a). Realistic dosimetric

111 applications, however, deal primarily with neutron fields that occupy one or several decades of

112 energy such that is necessary to generalize our expression for the ambient dose equivalent as

113

$$
H^{*}(10)=\int_{0}^{\infty} \Phi(E) h_{c c}(E) d E
$$

115 where $\Phi(E)$ contains the neutron energy characteristics (generally unknown) and $h_{c c}(E)$ is a 116 fluence-to-ambient dose equivalent conversion function. Note that $h_{c c}(E)$ is a highly nonlinear 117 function in energy wherein relatively low dose equivalent per unit neutron fluence ( $10 \mathrm{pSv}$ $118 \mathrm{~cm}^{2}$ ) is observed at energies below $10 \mathrm{keV}$ followed by a nearly two order-of-magnitude increase $119\left(\sim 600 \mathrm{pSv}-\mathrm{cm}^{2}\right)$ between $10 \mathrm{keV}$ and $1 \mathrm{MeV}$ as demonstrated in Figure 1a. This work will focus 120 on $h_{c c}(E)$ data presented by the International Committee on Radiological Protection (ICRP) in 121 publication $74[4]$.

123 Known neutron energy intensity as a function of axial or radial depth into a moderator (Figure

124 1b) permits the application of a Fredholm integral equation of the first kind as 125

$$
N(k)=\int_{0}^{\infty} R(E, k) \Phi(E) d E
$$


127 where $N(k)$ is the pulse height for energy bin $k$ with a known response matrix $R$. Such analyses

128 are commonly performed on Bonner sphere systems [2], utilizing multiple diameter spherical

129 moderators to provide different levels of thermalization for incident neutrons - each individual

130 moderator configuration corresponding to an exclusive, energy-dependent thermalization

131 efficiency curve that populates the $R(E, k)$ term (Fig. 1b). Neutrons that thermalize as they reach

132 the instrument's center can be detected and used to populate $N(k)$, thereby transforming

133 Equation (3) into an ill-posed, under-determined inversion problem [40] requiring a spectral

134 unfolding technique to determine $\Phi(E)$. Solution(s) obtained in this manner are not unique and

135 do not depend continuously on the data such that a more reliable, less computationally expensive

136 method is desirable for real-time dosimetric applications.
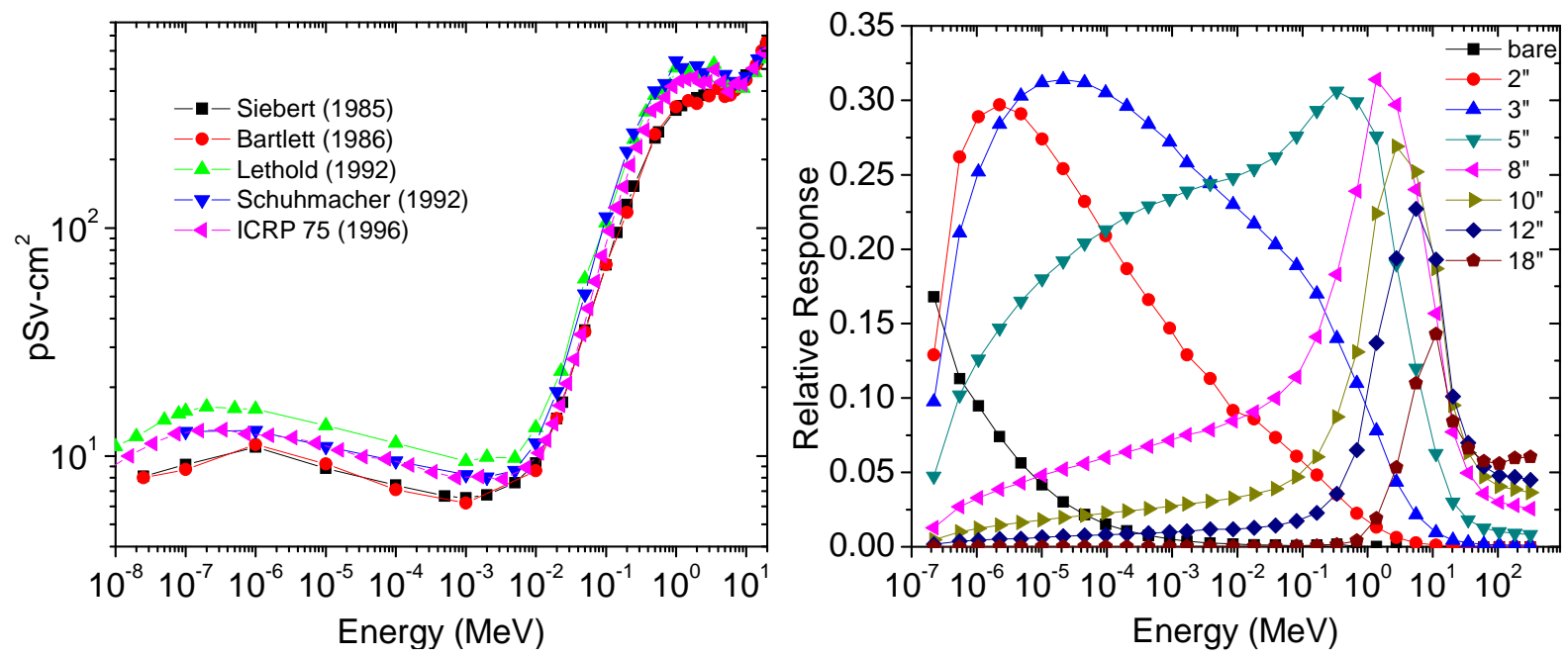

138 Fig. 1. (a) Various incarnations of the ambient dose equivalent conversion curve (- Siebert, •

139 Bartlett, $\Delta$ Lethold, $\boldsymbol{\nabla}$ Schuhmacher, 4 ICRP 74; adapted from [4, 41-45]); (b) response curves

140 from several Bonner sphere configurations.

142 The need for a portable, real-time neutron dose-equivalent meter was first addressed by Hankins

143 [1] in the form of a single thermal neutron detector surrounded by moderating material - 
144 essentially an adaptation of Bonner's spectrometer utilizing a single, fixed configuration. This

145 "rem meter" exhibits a measurement response

146

$$
M=\int_{0}^{\infty} C \Phi(E) d_{c c}(E) d E
$$

148 where $C$ is a calibration constant and $d_{c c}(E)$ is the energy-dependent detector response function.

149 Note the similarity in form between equations (2) and (4). Assuming that the neutron fields are

150 identical, it has been shown that matching the shape of a neutron detector's energy response

151 curve to the fluence-to-ambient dose equivalent conversion function provides an approximate

152 means of determining the neutron dose equivalent without the need to resolve the actual incident

153 energies [6]. A brief comparison of Figure $1 \mathrm{a}$ and $1 \mathrm{~b}$ enables the reader to infer the similarity in

154 shape between the response of the 10 to 18 " Bonner spheres and the ambient dose equivalent

155 coefficients up to $\sim 8 \mathrm{MeV}$. The resulting Andersson-Braun design (1963) and its variants (Fig. 2)

156 have been used to formulate several real-time devices including the SNOOPY (1964), LINUS

157 (1975), and WENDI-II (1995) [6-12]. 

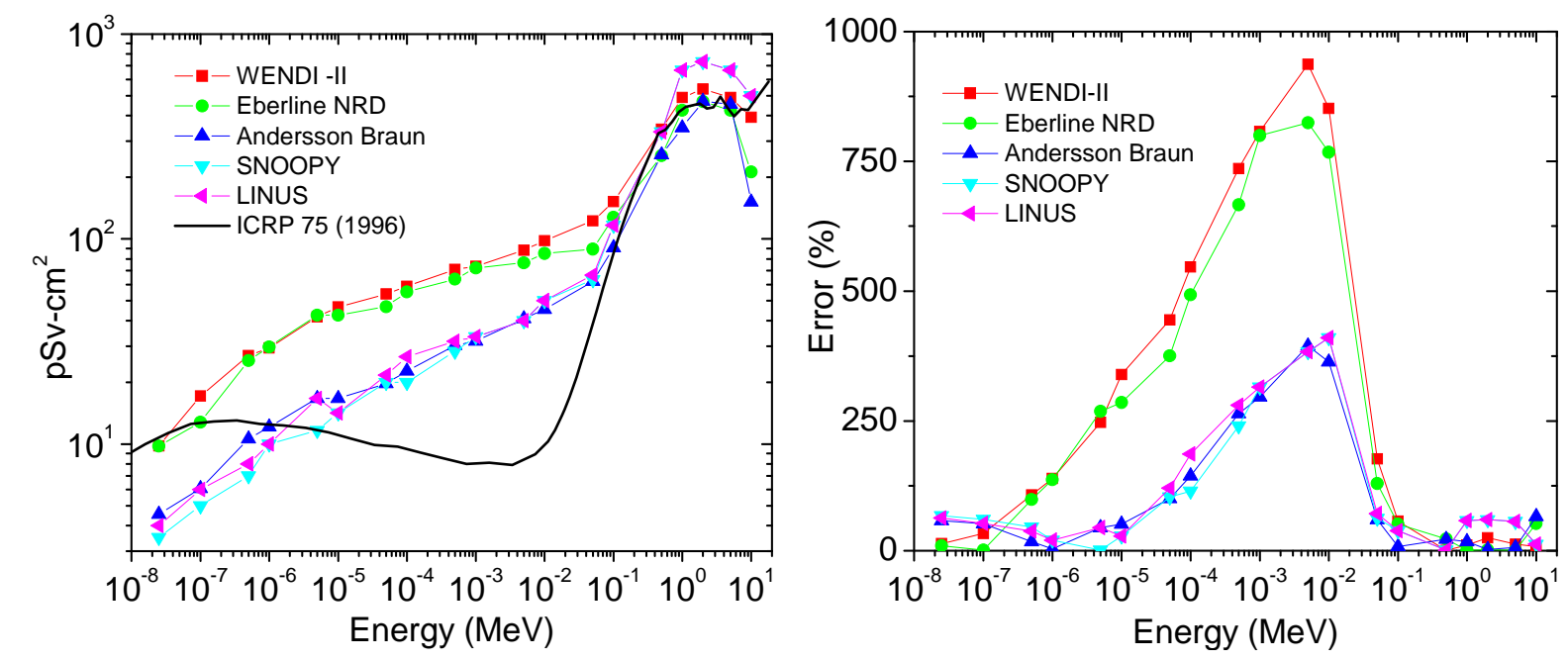

160 Fig. 2. (a) Calculated neutron-dose-equivalent energy-response for several neutron

161 detection/dosimetry models ( - WENDII-II, • Eberline NRD, $\boldsymbol{\Delta}$ Andersson Braun, $\nabla$ SNOOPY,

1624 LINUS, --- ICRP 74; adapted from [4, 6-12]); (b) and their associated error with respect to

163 ICRP 74 fluence-to-ambient dose equivalent conversion values.

165 Each of the detector responses shown in Figure 2 exhibit average errors ranging from 20 to 50

166 percent in the thermal and fast regions with considerable error present in the epithermal energy

167 range (i.e., $>950 \%$ of $h_{c c}(E)$ for the WENDI-II $[4,41-45]$ ). One may conclude that the accuracy

168 of such matching schemes is inherently limited by the use of a single detector and moderator

169 configuration.

171 In order to accurately match the non-linear shape of the ambient dose equivalent conversion

172 curve (or any future revisions that may result in its modification - Figure 1a) it is necessary - in

173 comparison with Bonner's work and as an improvement on the position sensitive long counter

174 [46] - to resolve (within $\sim 1 \mathrm{~cm}^{3}$ ) where incident neutrons reach thermal energy in a moderating

175 volume along one or more geometric coordinate axes. For the case of free neutrons travelling in 
176 parallel, this task can be accomplished by stacking high thermal efficiency solid state detectors

177 (or comparable thin high thermal efficiency detectors), into an axially symmetric moderator 178 geometry, like that of a right cylinder as shown by Figure 3a. "Thin" detectors are important as 179 they reduce the neutron scattering perturbation and reduce the total instrument volume. The $\sim 1$ $180 \mathrm{~cm}^{3}$ volume resolution recommendation is chosen as a volume that will yield fine enough 181 scattering length determination to the accurately quantify the neutron dose over many 182 logarithmic energy intervals. The volumetric or three-dimensional resolution comes from 183 stacking (1-D) pixelated (2-D) detectors. By doing so, not only can a real time response be 184 generated, but the conversion curve can also be adjusted electronically. Note, a non-pixilated 185 version, with stacking, that provides only 1-D resolution along the axial coordinate of a cylinder 186 is also possible. Further, it is possible to replace the solid-state detectors, as long as the replacing 187 detector(s) is/are comparably low volume relative to the overall volume and has (or can be 188 summed to provide) at least one-dimensional position sensitivity. In the case of the instrument 189 described here, it is assumed that the neutrons are parallel and incident on the front face of the 190 right cylinder as shown in Figure 3a. In applications with significant scattering, the instrument 191 would be covered by a material that absorbs thermal neutrons, such as cadmium or a boron 192 compound, and the absorbing layer covered with moderator to avoid detecting epithermal and 193 fast neutrons from the sides or back (i.e. a camera geometry). Conversely, if there were very few 194 neutrons and they were incident from all directions, a spherical geometry with radial dependence 195 would be optimal. For the instrument described henceforth, the discussion is focused on the 1-D 196 version (i.e., axial dependence) of the cylindrical geometry wherein $n$ neutron detectors are 197 stacked at $1.0 \mathrm{~cm}$ center-to-center spacing and oriented to maintain axial symmetry within a 198 hydrogenous moderator of comparable radius (Figures 3a and 4a). Moderator length (axial 
199 dimension) is chosen in consideration of the scattering length needed to accurately resolve the 200 dose of $15 \mathrm{MeV}$ neutrons (i.e. $\sim 15.0 \mathrm{~cm}$ ).

201

202
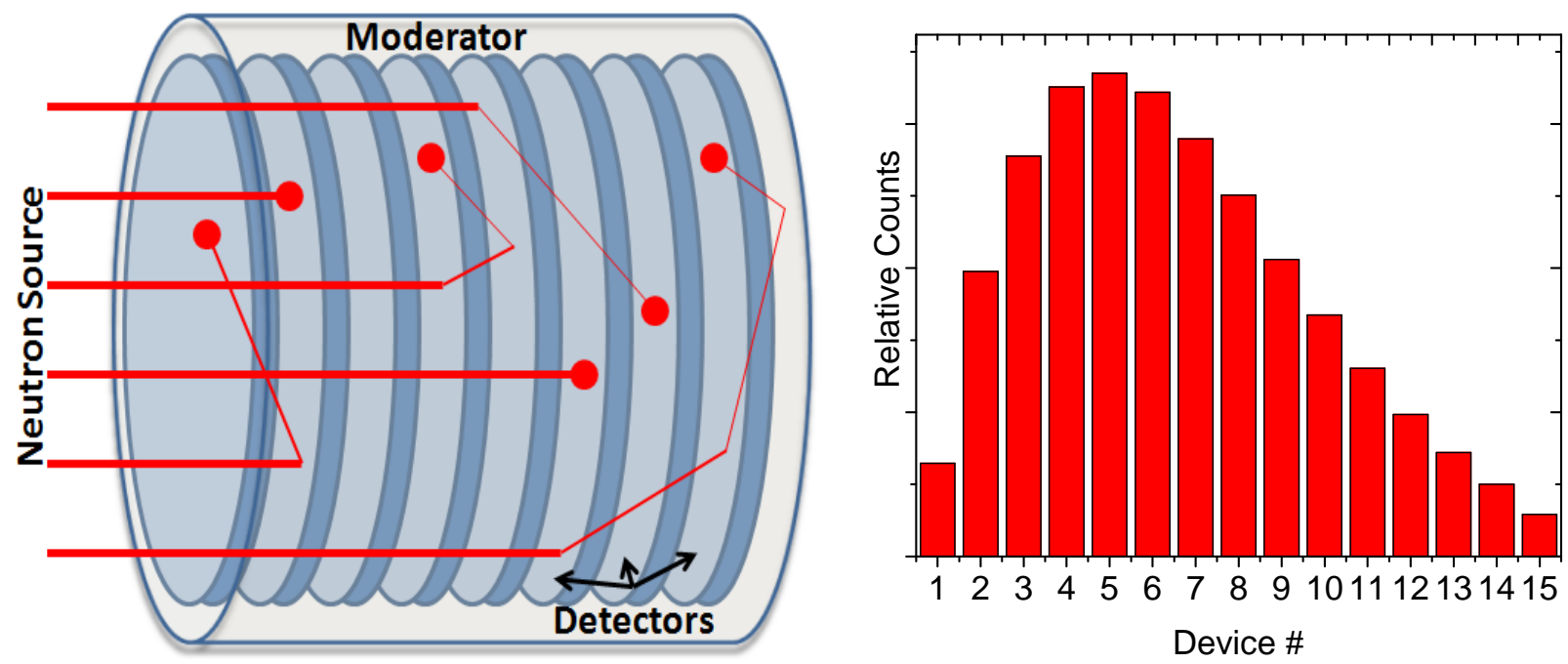

203 Fig. 3. (a) Adaptation of the Bonner Sphere system into a cylindrical symmetry with solid state

204 thermal neutron detectors allowing for simultaneous detector response as a function of the axial

205 dimension; (b) histogram tallies of measured counts (point of thermalization) from bare ${ }^{252} \mathrm{Cf}$

$206[48]$ as a function of axial position into the moderator.

207

208 The 1-D axial binning scheme is presented in the form of a histogram in Figure 3b, unique to the

209 energy and intensity of the incident neutron source (unmoderated ${ }^{252} \mathrm{Cf}$ in this case). The

210 thickness/volume of a solid-state detector is defined by the semiconductor element and any

211 necessary electronics that must be in the neutron path (e.g., preamplifiers, fiberglass boards,

212 etc.). One means of meeting the needed specifications for thermal efficiency, large area and low

213 volume (i.e., thin) are the indirect-conversion, solid state neutron detectors developed at Kansas

214 State University [37]. These microstructured neutron detectors (MSNDs) are comprised of

215 silicon micro-structural trenches, doped and contacted to enable a p-n junction, and backfilled 
216 with enriched ${ }^{6} \mathrm{LiF}$ powder. The microstructure dimensions and lower level discriminator settings

217 have been optimized for the ${ }^{6} \mathrm{Li}$ primary reaction products mean free paths to yield devices with

$21822 \%$ thermal neutron detection efficiency. Because standard VLSI methods are used to process

219 the MSNDs, device radii in excess of $10 \mathrm{~cm}$ - built either from a single $200 \mathrm{~mm}$ wafer or from

220 the superposition of wafer slices from $125 \mathrm{~mm}$ wafers - are possible and explored as an upper

221 bound in the calculations described below.

222

223 The minimal perturbation of each detector to the moderation process, combined with the high

224 thermal efficiency of each solid-state element, permits the investigation of an individual device's

225 output with respect to the corresponding degree of observed moderator penetration. Energy

226 dependence considerations allow for the delivery of distinct efficiency vs. energy curves as a

227 function of moderator thickness that closely resembles the acquisition from collections of Bonner

228 sphere configurations (Fig. 1a) - but in real time and without the significant non-detectable

229 absorption that occurs in the Bonner Sphere and related instruments. The availability of $n$

230 simultaneous measurements from $n$ detectors with unique, Bonner-like response functions

231 permits revision of its rem meter's dose response curve to

232

$$
M=\int_{0}^{\infty} \Phi(E) f\left(d_{c c, 1}(E), \ldots, d_{c c, n}(E)\right) d E
$$

234 where the single detector response curve of a conventional rem meter is replaced by some 235 function, $f$, of multiple response curves, $d_{c c, 1}(E)-d_{c c, n}(E)$, to permit more accurate matching to $236 h_{c c}(E)$. It is proposed that a linear combination of the individual Bonner-like response functions 
237 can be used to force the rem meter's overall response function to mimic the shape of the provided

238 fluence-to-ambient dose equivalent conversion function such that

239

$$
f\left(d_{c c, 1}(E), \ldots, d_{c c, n}(E)\right)=h_{c c}(E)=\sum_{i=1}^{n} g_{i} d_{c c, i}(E)
$$

241 where $g_{i}$ is the gain corresponding to the $i_{t h}$ detector's response function. It is this gain that

242 allows for the electronic matching to any dose equivalent curve. A collection of measurements

243 from $m$ mono-energetic sources spanning the pertinent energy range are required to populate an

$244 m$ by $n$ matrix, $B$, where the corresponding $h_{c c}(E)$ values populate a $m$ by 1 column matrix, $y$.

245 The discrete Fredholm equation is then expressed as

246

$y_{(m, 1)}=B_{(m, n)} G_{(n, 1)}$

248 where $G$ is the gain matrix containing $n$ optimal multiplier values $\left(g_{1}-g_{n}\right)$. Assuming an over-

249 determined system, identification of the optimal gain values is now accomplished by 250 minimization of a "cost" function, selected for this case to be the sum of the square of the 251 residuals

252

$J=\left[y_{(m, 1)}-B_{(m, n)} G_{(n, 1)}\right]^{T} R_{(m, m)}{ }^{-1}\left[y_{(m, 1)}-B_{(m, n)} G_{(m, 1)}\right]$

254 where $R$ is a diagonal matrix populated by the desired weights, for this case the inverse square 255 values of $y$ [47]. Assuming $B$ is invertible 
$G_{(n, 1)}=\left[B_{(n, m)}^{T} R_{(m, m)}^{-1} B_{(m, n)}\right]^{-1} B_{(n, m)}^{T} R_{(m, m)}^{-1} y_{(m, 1)}$

258 Once the gain values are determined, the ambient dose equivalent due to a cumulative detector

259 response (i.e. $n$ detectors) can be determined from a series of backward substitutions as

260

$H^{*}(10)=\sum_{i=1}^{n} g_{i} M_{i}(\mu \mathrm{Sv})$

262 where $M_{i}$ denotes the number of counts on the $i^{\text {th }}$ detector, or

263

$M_{i}=\int_{0}^{\infty} \Phi(E) d_{c c, i}(E) d E$

\section{Computational Modeling}

266 Instrument studies were performed using the Monte Carlo N-Particle code (MCNP), specifically

267 MCNPX 2.6.0 for charged particle transport. All experiments conducted in the current study

268 utilize a similar, high-density polyethylene moderated model (Fig. 4a) with simulations driven by

269 a planar source of 5000 neutrons per $\mathrm{cm}^{2}-$ in all cases the source radius is set equal to the

270 detector/moderator radius. Neutron detectors are modeled as $525 \mu$ m-thick cylinders of natural

271 isotopic abundance silicon containing homogeneously interspersed quantities of neutron-

272 sensitive material sufficient enough to yield $22 \%$ thermal detection efficiency, commensurate

273 with the efficiency obtained with the ${ }^{6} \mathrm{LiF}$ solid state detectors developed at Kansas State 
274 University [37]. Alpha production in each transduction cell is accounted via series of $\mathrm{f} 4$ tallies

275 where a one-to-one ratio exists between realized alpha particles and successfully detected

276 neutrons per the cell material definition. Three sets of primary simulations are conducted on a

277 generalized MCNPX model (Fig. 4a) with $\mathrm{L}=15.0 \mathrm{~cm}$ for detector radii of 5.0, 7.0, and $10.0 \mathrm{~cm}$,

278 the latter combination corresponding to a maximum desired moderator mass of $4.5 \mathrm{~kg}$. Each set

279 features a collection of 23 different mono-energetic neutron sources spaced logarithmically

280 between $10^{-8}$ and $15 \mathrm{MeV}$ with the results compiled into output histograms (one per simulation;

281 see Figure $3 b$ for an example).

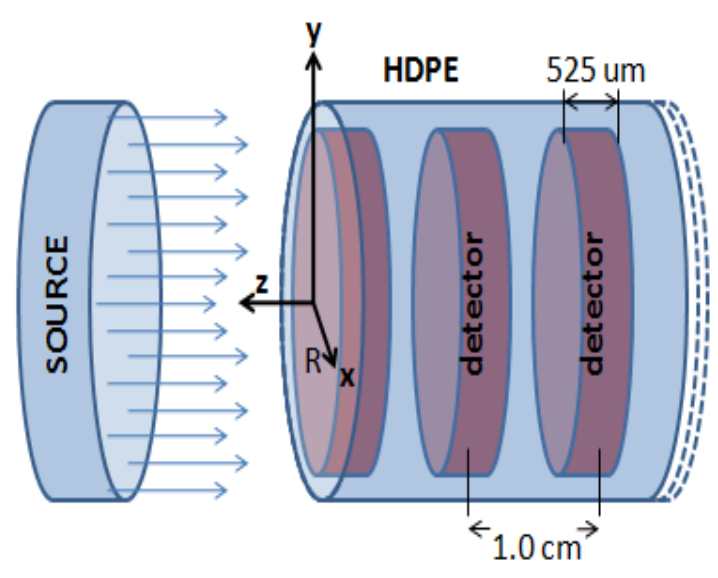

282

283

284

285

286

287

288

289

290 configuration.

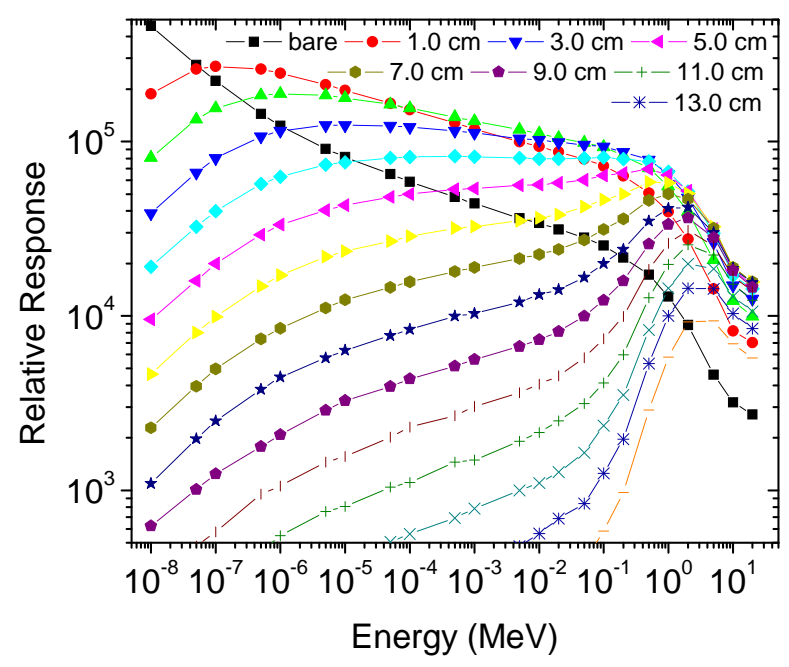

Fig. 4. (a) Generalized MCNPX model schematic for the solid state neutron spectrometer reported here; (b) detector position specific response curves for the $r=10.0 . \mathrm{cm}, L=15.0 \mathrm{~cm}$

Higher kinetic energy neutrons exhibit larger total path lengths between scattering interactions needed to reach thermal energy, and are therefore capable of further axial penetrations into the detector. This phenomenon yields count distributions (intensity as a function of axial position) that feature markedly different uni-modal shapes as a function of energy. Tabulation of the 
291 histogram collections permits presentation of the individual device efficiencies as a function of 292 neutron energy (Fig. 4b) that closely resemble the outputs of different Bonner sphere 293 configurations. Note that while the shape remains consistent between the different models, the 294 calculated values appear higher in all cases for larger volume detectors $(10.0 \mathrm{~cm}>7.0 \mathrm{~cm}>5.0$ $295 \mathrm{~cm})$ likely due to the subsequent increase in the relative number of probable scattering reactions 296 (i.e. intrinsic efficiency).
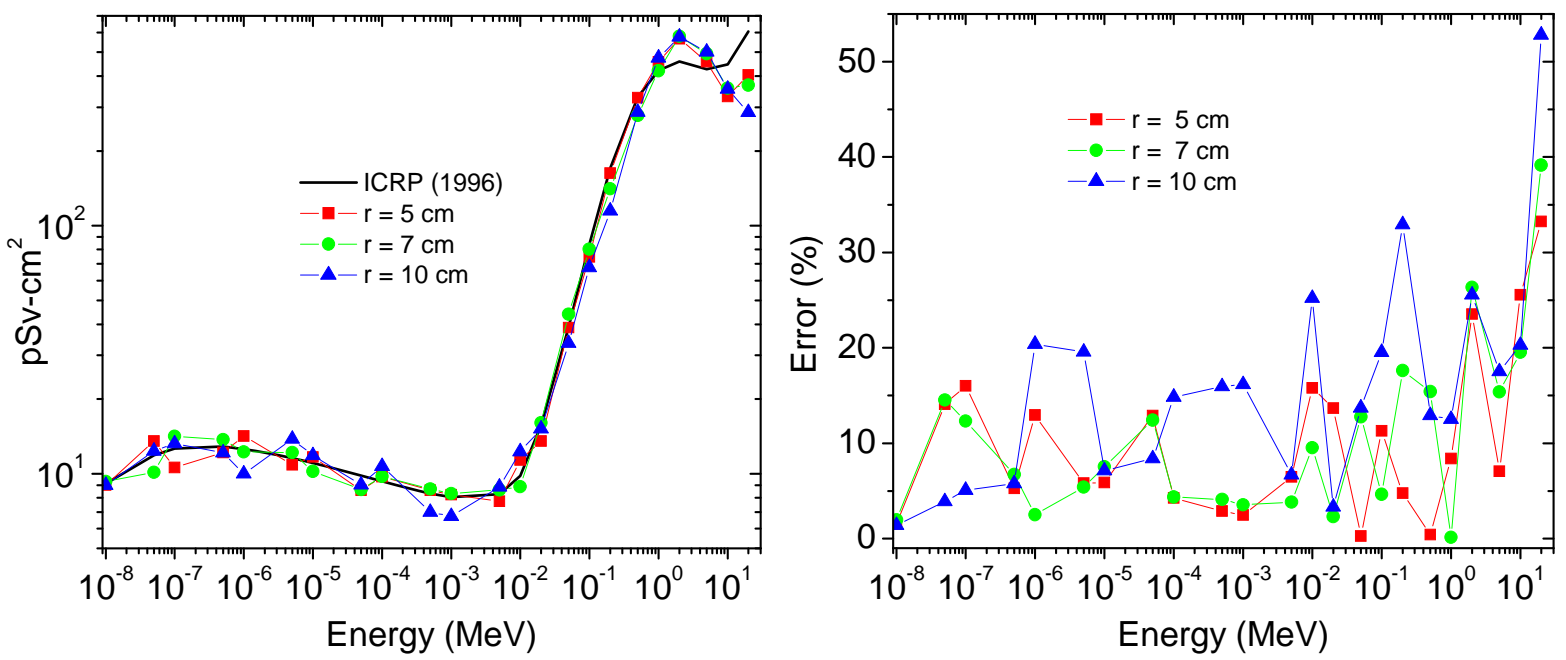

299 Fig. 5. Response (a) and error (b) of the instrument reported here for $r=5(\bullet), 7(\bullet), 10(\Delta) \mathrm{cm}$

300 and $L=15 \mathrm{~cm}$. The instrument response in (a) is compared to ICRP 74 fluence-to-ambient dose

301 equivalent conversion values.

302

303 Equations (6) - (10) are used in conjunction with the data acquired from each simulation set to 304 match the detector response function to the reference $h_{c c}(E)$ curve (Fig. 5) where $n=15$ and $m=$ 30523 (15 devices and 23 appropriately spaced mono-energetic simulations). As shown in Figure 5a, 306 each of the dosimeter radii exhibit excellent tracking of the reference $h_{c c}(E)$ curve in the range 307 of thermal to $20 \mathrm{MeV}$. The average errors over the entire energy span measure 10.2, 10.5, and 
30815.7 percent, respectively, with the absolute maximums observed between 15 and $20 \mathrm{MeV}$ for all

309 cases. These errors are significantly less than those of conventional rem meters displayed in

310 Figure 2. In addition, the three proposed dosimeters evaluated here have moderator masses of

311 only $1.1,2.3$, or $4.5 \mathrm{~kg}$, depending on the radius utilized. In environments where scattered

312 neutrons may impinge on the side or back of the instrument, the concentric cadmium wrapping

313 and moderator (assuming $\sim 3.0 \mathrm{~cm}$ thickness to appropriately thermalize most epithermal

314 neutrons prior to passage through the cadmium layer) will add $1.7,2.2$ or $3.0 \mathrm{~kg}$ to the total

315 instrument mass.

316

317 4. Model Validation and Discussion

318 Validation of the computed ambient dose equivalent is accomplished through superposition of 319 data sets collected from the 23 monoenergetic neutron simulations in section 3 to emulate four 320 different neutron energy distributions: the first two constructed from the neutron spectra arising 321 from the AmBe and ${ }^{252} \mathrm{Cf}$ sources (Fig. 6a [48]), the third from an unrealistic, entirely epithermal 322 energy range, and the fourth from equal dose contributions of thermal, epithermal, and fast 323 neutrons (Fig. 6b). The individual contributions from each simulation histogram/energy are 324 modified to deliver a net dose of $10 \mu \mathrm{Sv}(1.0 \mathrm{mrem})$. 

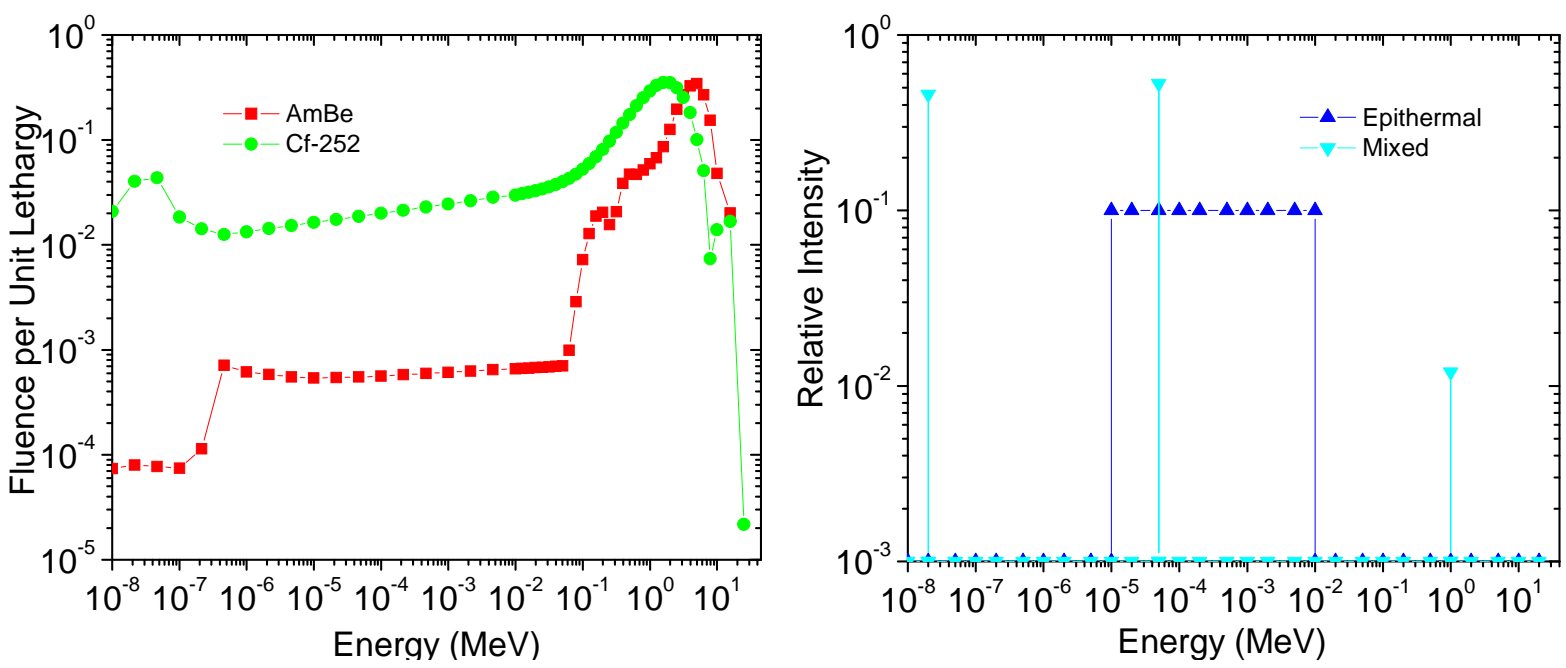

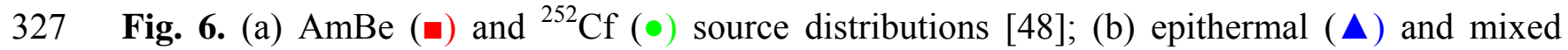
328 monoenergetic $(\nabla)$ source distributions.

330 The histogram data provided by each simulation output is used in conjunction with equation (10)

331 to estimate the ambient dose equivalent (Table 1). All of the models/estimates accurately account

332 for the delivered equivalent dose with all observed errors less than $15 \%$ for all cases (energy and 333 radii).

\begin{tabular}{|c|c|c|c|c|}
\hline Model/Source & AmBe (\%) & ${ }^{252} \mathbf{C f}$ (\%) & $\begin{array}{c}\text { Epithermal } \\
(\%)\end{array}$ & $\begin{array}{c}\text { Mixed Mono } \\
\text { (\%) }\end{array}$ \\
\hline $\mathbf{R}=\mathbf{5 . 0} \mathbf{~ c m}$ & 7.5 & 11.7 & 2.2 & 0.1 \\
\hline $\mathbf{R}=\mathbf{7 . 0} \mathbf{~ c m}$ & 11.3 & 8.8 & 4.0 & 0.9 \\
\hline $\mathbf{R}=\mathbf{1 0 . 0} \mathbf{~ c m}$ & 13.3 & 12.0 & 1.0 & 0.9 \\
\hline
\end{tabular}

335 Table 1: Error in estimation of reference dose equivalent for neutron source distributions. 
337 Note that most of this error is observed in the $\mathrm{AmBe}$ and ${ }^{252} \mathrm{Cf}$ spectra and may be attributed to 338 the fact that the majority of their respective dose contributions are derived from higher energy 339 neutrons where the greatest disparity between $h_{c c}(E)$ and instrument response is observed.

340 Conversely, the doses delivered by epithermal and mixed mono-energetic neutron sources 341 exhibit measurement errors less than $4 \%$ and speak directly to the accurate response-matching at

342 energies below 1.0 MeV. Further enhancement to response-matching is likely attainable via 343 design optimization (i.e. different length, radius, detector spacing, etc.) in conjunction with 344 subsequent improvements to equation (6) (i.e. perhaps a more complicated function of the 345 different response curves). Further, it is important to note that the current form of equation (6) 346 permits both positive and negative multipliers which, with poor counting statistics, could lead to 347 erroneous dose estimates. Although poor counting statistics are mitigated by the high neutron 348 efficiency of this device, this effect will be addressed in future work.

350 In addition to size, mass, and energy-response characteristics, a rem meter's measurement 351 sensitivity and/or intrinsic efficiency must also be considered when evaluating its overall 352 performance. Canberra's NP2 SNOOPY - an $11.8 \mathrm{~kg}$ instrument commonly used for dosimetric 353 surveys of reactor spectra - features a lateral sensitivity of $\sim 10.0$ counts/minute per $\mu \mathrm{Sv} /$ hour 354 referenced to ${ }^{252} \mathrm{Cf}$. Assuming a total side-irradiation $(24.38 \mathrm{~cm}$ by $40.64 \mathrm{~cm})$ and $380 \mathrm{pSv}-\mathrm{cm} 2$ 355 average dose-equivalent per unit-neutron-fluence [48], this translates to $0.05 \%$ intrinsic 356 efficiency. Despite errors upward of $400 \%$ in the epithermal energy region, the SNOOPY 357 reportedly maintains $10 \%$ uncertainty with respect to reference dosimetric values (likely due to 358 the generally mid-to-high-range energy spectra to which it is intended to encounter); however, as 
many real world neutron fields comprise a significant scattering fraction, accurately resolving the

360 epithermal neutron dose equivalent cannot be ignored.

362 Thermo's WENDI-II incorporates the addition of spallation material (i.e. lead) that extends its 363 energy range upwards of $5.0 \mathrm{GeV}$ for monitoring neutron fields resulting from high-energy

364 accelerators and/or cosmic interactions. The spallation centers consequently increases the total 365 mass to $13.2 \mathrm{~kg}$ and increases the epithermal error above $900 \%$. It too maintains a $10 \%$ 366 uncertainty to unmoderated spontaneous-fission- or $\alpha, n$-spectrum-type doses - most likely due to

367 its accurate matching of the dose-equivalent curve at energies greater than $1.0 \mathrm{MeV}-$ and 368 exhibits a lateral sensitivity approximately five times greater than that of the SNOOPY (45.7 369 counts/minute per $\mu \mathrm{Sv} /$ hour $)$. Given the similar dimensions $(22.86 \mathrm{~cm}$ by $33.67 \mathrm{~cm})$ between the 370 two devices, this increase in measurement sensitivity directly corresponds to a five-fold increase 371 in observed intrinsic efficiency to $0.25 \%$.

372

373 In contrast to conventional neutron dose-equivalent survey technology, the instrument reported 374 here permits dose-equivalent measurements in the energy range of thermal to $20.0 \mathrm{MeV}$ within $37515 \%$ accuracy over the total range with less than half of the required mass. All three simulated 376 systems exhibit intrinsic efficiencies to bare ${ }^{252} \mathrm{Cf}$ of $10.25 \%, 18.89 \%$, and $27.70 \%$ (for $r=5,7$, 377 and $10 \mathrm{~cm}$, respectively) and measurement sensitivities in terms of raw count data of $353,6,750$, 378 and 13,780 counts/minute per $\mu \mathrm{Sv} /$ hour (for $r=5,7$, and $10 \mathrm{~cm}$, respectively). This significant 379 increase in instrument sensitivity/intrinsic efficiency related to the SNOOPY or WENDI-II is 380 based on the presence of high thermal efficiency detectors distributed $1 \mathrm{~cm}$ along the 381 thermalization path which permit detection of neutrons that are otherwise lost to capture in 
382 traditional instruments with a $12 \mathrm{~cm}$ moderator radius and single central detector. In

383 concurrence, is important to note that the sensitivities and intrinsic efficiencies of the system

384 described here, solely associated with the deepest detectors, are comparable with those of the

385 SNOOPY and WENDI-II systems (i.e., $\sim 0.25 \%$ ).

\section{5. Summary and Future}

388 A new type of portable neutron rem meter is introduced based on the concept of a solid state 389 neutron spectrometer. The instrument design and algorithm developed are motivated by the high 390 error encountered with commercially available wide-energy range neutron dose equivalent

391 instruments. The device utilizes real-time sampling of thermalized neutrons by multiple weakly 392 perturbing and high thermal efficiency solid-state neutron detectors to provide simultaneous 393 access to a number of Bonner-like response curves. A linear combination of the measurement 394 signals permits excellent matching of the energy-dependent ambient dose equivalent coefficients 395 with average errors less than $15 \%$. Validation of the measured ambient equivalent neutron dose 396 is accomplished using simulation-compiled $\mathrm{AmBe},{ }^{252} \mathrm{Cf}$, epithermal, and mixed mono-energetic 397 spectra to yield absolute errors less than 15\% for all cases. These investigations have yet to 398 consider the propagation of counting statistics on individual detectors to the resulting dose 399 prediction that will be needed to confirm dosimetry accuracy for low flux neutron dose fields 400 and/or short counting times in the 15 second range typically associated with practical neutron 401 dose survey meter applications.

402

403 Acknowledgements 
404 The authors acknowledge partial support of this work by the Office of Naval Research under

405 award No. N00014-11-1-0157. ANC wishes to thank Noel Guardala for helpful discussions in

406 originating this work.

\section{References}

[1] Dale E. Hankins, Report LA-2717, Los Alamos Scientific Laboratory, Los Alamos (1962).

[2] R.L. Bramblett, R.J. Ewing and T.W. Bonner, Nucl. Instrum. Methods 9 (1960) 1.

[3] ICRP, 2007. The 2007 Recommendations of the International Commission on Radiological Protection. ICRP Publication 103. Ann. ICRP 37 (2-4).

[4] ICRP, Conversion Coefficients for Use in Radiological Protection against External Radiation, Publication 74, International Commission on Radiological Protection, Annals of the ICRP, 26, No. 3/4, Pergamon Press, Oxford, 1996.

[5] J.C. McDonald, B.R.L. Siebert, W.G. Alberts, Nuc. Inst. Meth. Phys. Res. A 476 (2002) 347.

[6] Richard H. Olsher, Hsiao-Hua Hsu, Anthony Beverding, Jeffrey H. Kleck, William H. Casson, Dinnis G. Vasilik, Robert T. Devine, Health Physics 79 (2000) 170.

[7] V. Mares, A.V. Sannikov, H. Schraube, Nuc. Inst. Meth. Phys. Res. A 476 (2002) 341.

[8] C. Birattari, A. Ferrari, C. Nuccetelli, M. Pelliccioni, M. Silari, Nuc. Inst. Meth. Phys. Res. A 297 (1990) 250.

[9] J.M. Brushwood, P.A. Beeley, N.M. Spyrou, Nuc. Inst. Meth. Phys. Res. A 476 (2002) 304.

[10] Chris Benson, Malcolm J. Joyce, Barry O-Connell, Jon Silvie, IEEE Trans. Nuc. Sci. 47 (2000) 2417.

[11] M. Cosack, H. Lesiecki, Rad. Prot. Dos. 10 (1985) 111.

[12] J.W. Leake, Nuc. Inst. Meth. 45 (1966) 151.

[13] J.A. Weaver, M.J. Joyce, A.J. Peyton, J. Roskell, M.J. Armishaw, Rev. Sci. Inst. 72 (2001) 2043.

[14] J.A. Weaver, M.J. Joyce, A.J. Peyton, J. Roskell, Nuc. Inst. Meth. Phys. Res. A 476 (2002) 143. 
[15] A.M. Williams, N.M. Spyrou, J.M. Brushwood, P.A. Beeley, Nuc. Inst. Meth. Phys. Res. A 476 (2002) 149.

[16] Gordon K. Riel, Lightweight Neutron Remmeter, United States Patent No. 6,930,311 (2005).

[17] R. Olsher, D. Seagraves, S. Eisele, C. Bjork, W. Martinez, L. Romero, M. Mallett, M. Duran. C. Hurlbut, "Prescila: A New, Lightweight Neutron Rem Meter," Health Physics 86 (2004) 603-612.

[18] J. Pope, Radiat. Prot. Management 11 (1994), 91-96.

[19] H. Toyokawa, A. Uritani, C. Mori, N. Takeda, K. Kudo, IEEE Trans. Nuc. Sci. 42 (1995) 644.

[20] H. Toyokawa, A. Uritani, C. Mori, M. Yoshizawa, N. Takeda, K. Kudo, Nuc. Inst. Meth. Phys. Res. A 381 (1996) 481.

[21] H. Toyokawa, M. Yoshizawa, A. Uritani, C. Mori, N. Takeda, K. Kudo, IEEE Trans. Nuc. Sci. 44 (1997) 788.

[22] H. Toyokawa, A. Uritani, C. Mori, N. Takeda, K. Kudo, Rad. Prot. Dos. 70 (1997) 365.

[23] S. Yamaguchi, A. Uritani, H. Sakai, C. Mori, T. Iguchi, H. Toyokawa, N. Takeda, K. Kudo, Nuc. Inst. Meth. Phys. Res. A 422 (1999) 600.

[24] R.J. Sheu, J.S. Lin, S.H. Jiang, Nuc. Inst. Meth. Phys. Res. A 476 (2002) 74.

[25] J.L. Muniz, M.C. Vincente, E.M. Gonzalez, A.M. Romero, M. Embid, A. Delgado, Rad. Prot. Dos. 110 (2004) 243.

[26] Taosheng Li, Lianzhen Yang, Jizeng Ma, Dong Fang, Rad. Prot. Dos. 123 (2007) 15.

[27] Andrew C. Stephan and Vincent D. Jardret, Neutron Detector, United States Patent No. 7,514,694 (2009).

[28] Stephen H. Manglos, Neutron Range Spectrometer, United States Patent No. 4,837,442 (1989).

[29] Garry B. Spector, Tom McCollum, Alexander R. Spowart, Nuc. Inst. Meth. Phys. Res. A 346 (1994) 273.

[30] Y. Tanimura, J. Saegusa, M. Yoshizawa, M. Yoshida, Nuc. Inst. Meth. Phys. Res. A 547 (2005) 592. 
[31] Tom McCollum, Scintillator Fiber Optic Long Counter, United States Patent No. $5,298,756$ (1994).

[32] D.T. Bartlett, R.J. Tanner, D.G. Jones, Rad. Prot. Dos. 74 (1997) 267.

[33] M.J. Joyce, B.R. More, D.T. Bartlett, R.J. Tanner, D.G. Jones, Neutron Radiation Detector, United States Patent No. 6,362,485 (2002).

[34] S.D. Monk, M.J. Joyce, Rad. Prot. Dos. 123 (2007) 3.

[35] S.D. Monk, M.J. Joyce, Z. Jarrah, D. King, M. Oppenheim, Rev. Sci. Inst. 79 (2008) 023301.

[36] A.N. Caruso, J. Phys.: Cond. Matt. 22 (2010) 443201.

[37] D.S. McGregor, W.J. McNeil, S.L. Bellinger, T.C. Unruh, J.K. Shultis, Nuc. Inst. Meth. Phys. Res. A 608 (2009) 125.

[38] T.M. Oakes, "Modeling and Analysis of a Portable, Solid-State Neutron Detection System for Spectroscopic Applications", PhD Dissertation, 2012.

[39] ICRP, 1990 Recommendations of the International Commission for Radiological Protection, Publication 60, International Commission on Radiological Protection, Annals of the ICRP, 23, Pergamon Press, Oxford, 1991.

[40] Y. Xu, M. Flaska, S. Pozzi, V. Protopopescu, T. Downar, Proc. of Joint Intl. Top. Meet.: $M \& C+S N A$ (2007) Monterey, California.

[41] A. Ferrari, M. Pelliccioni, Rad. Prot. Dos. 76 (1998) 215.

[42] H. Schuhmacher, B. Siebert, Rad. Prot. Dos. 40 (1992) 85.

[43] D. Bartlett, Rad. Prot. Dos. 15 (1986) 273.

[44] B. Siebert, R. Hollnagel, Rad. Prot. Dos. 12 (1985) 145.

[45] G. Leuthold, V. Mares, H. Schraube, Rad. Prot. Dos. 40 (1992) 77.

[46] Y. Tanimura, J. Saegusa, M. Yoshizawa, M. Yoshida, Nuc. Inst. Meth. Phys. Res. A 346 (1994) 273-278.

[47] Simon, Dan, "Optimal State Estimation: Kalman, $\mathrm{H}_{\infty}$, and Nonlinear Approaches," Hoboken, New Jersey: John Wiley \& Sons, 2006. 
537

538

539

[48] IAEA, Compendium of neutron spectra and detector responses for radiation protection purposes. Technical Reports Series No. 403, Supplement to Technical Reports Series No. 318. Vienna (2001). 